\title{
Measuring and Understanding the Universe
}

\author{
Wendy L. Freedman* \\ Observatories of the Carnegie Institution of Washington, 813 Santa Barbara St., \\ Pasadena, CA 91101, USA \\ Michael S. Turner \\ Center for Cosmological Physics and the Departments of Astronomy 8 Astrophysics \\ and of Physics, The University of Chicago, 5640 S. Ellis Ave., Chicago, \\ IL 60637-1433 and NASA/Fermilab Astrophysics Center, Fermi National \\ Accelerator Laboratory, MS 209, PO Box 500, Batavia, IL 60510-0500
}

\begin{abstract}
Revolutionary advances in both theory and technology have launched cosmology into its most exciting period of discovery yet. Unanticipated components of the universe have been identified, promising ideas for understanding the basic features of the universe are being tested, and deep connections between physics on the smallest scales and on the largest scales are being revealed.
\end{abstract}

*Electronic address: wendy@ociw.edu 


\section{Contents}

I. Introduction

II. TAKING THE MEASURE OF THE UNIVERSE

A. Cosmological Framework 4

B. The Expansion of the Universe $\quad 6$

C. The Matter Composition of the Universe 1

D. The Cosmic Microwave Background 10

E. The Structure of the Universe Todav 12

F. The Age of the Universe 14

G. Recap: The New Emerging Cosmology

III. UNDERSTANDING THE UNIVERSE

$\begin{array}{ll}\text { A. Origin of Structure } & 15\end{array}$

B. The Expansion 17

$\begin{array}{ll}\text { C. The Composition of the universe } & 20\end{array}$

IV. LOOKING FORWARD 22

V. WMAP Postscript 23

References

$\begin{array}{ll}\text { Figures } & 27\end{array}$

Tables

\section{INTRODUCTION}

Thirty years ago, cosmology was described as a search for two numbers: the current expansion rate (or Hubble constant), $H_{0}$, and its change over time, the deceleration parameter, $q_{0}$ (Sandage, 1970). But that was before the discovery of giant walls of galaxies, voids, dark matter, tiny variations in the cosmic microwave background radiation (CMB), dark energy and the acceleration of the Universe. Today, the subject has become vastly richer, and the 
numbers being sought are more numerous but more closely tied to fundamental theory. An overall picture has emerged that accounts for the origin of structure and geometry of the Universe, as well as describing its evolution from a fraction of a second onward.

In this new and still-evolving picture rooted in elementary particle physics, in a tiny fraction of a second during the early history of the universe, there was an enormous expansion called inflation. This expansion smoothed out wrinkles and curvature in the fabric of spacetime, and stretched quantum fluctuations on subatomic scales to astrophysical scales. Following inflation was a phase when the Universe was a hot thermal mixture of elementary particles, out of which arose all the forms of matter that exist today. Some 10,000 years into its evolution, gravity began to grow the tiny lumpiness in the matter distribution arising from quantum fluctuations into the rich cosmic structures seen today, from individual galaxies to the great clusters of galaxies and superclusters.

Recent observations of the universe have not only strengthened and expanded the bigbang model, but they have also revealed surprises. In particular, most of the universe is made of something fundamentally different from the ordinary matter we are made of. (By ordinary matter, we mean matter made of neutrons and protons; the jargon for this is baryons, the technical term for particles made of quark triplets.) About 30\% of the total mass-energy is dark matter, composed of particles most likely formed early in the universe. Two thirds is in a smooth "dark energy" whose gravitational effects began causing the expansion of the universe to speed up just a few billion years ago. Ordinary matter, the bulk of it dark, only accounts for the remaining $4 \%$ of the total mass-energy density of the universe. While the remnant (thermal) microwave background from the hot big bang contributes only about $0.01 \%$, it encodes information about the spacetime structure of the Universe, about its early history, and possibly even about its ultimate fate.

We have also learned much about the organization of the universe. In the nearby universe, galaxies are distributed in a "cosmic web" composed of sheets and sinuous filaments interspersed with voids (see Figures 1 and 2). Though inhomogeneous on these apparently vast scales, the Universe becomes more and more homogeneous when viewed on even larger scales from $100 \mathrm{Mpc}$ out to the current horizon of 10,000 Mpc.

In the first part of this review, we describe the universe - its structure, composition and global properties. Then we proceed to discuss our current understanding of its origin and early evolution, emphasizing the deep connections between physics on the smallest and 
largest scales. We end by discussing some recent and more speculative ideas from theory, as well as posing some of the "big questions" confronting cosmology today.

\section{TAKING THE MEASURE OF THE UNIVERSE}

\section{A. Cosmological Framework}

The framework for understanding the evolution of the universe is the hot big-bang model, technically referred to as the Friedmann-Lemaitre-Robertson-Walker (FLRW) cosmological model. Grounded in Einstein's theory of general relativity, this model assumes that on the largest scales the universe is homogeneous and isotropic, features which have now been confirmed observationally.

The FLRW model incorporating inflation, can be described by 16 cosmological parameters that we group here into two categories (see Table I). The first 10 parameters describe the expansion, the global geometry, the age and the composition of the underlying FLRW model, while the final 6 describe the deviations from exact homogeneity, which at early times were small, but today manifest themselves in the abundance of cosmic structure, from galaxies to superclusters.

The Friedmann equation governs the expansion rate and relates several of the first 10 parameters:

$$
\begin{aligned}
H^{2} \equiv\left(\frac{\dot{a}}{a}\right)^{2} & =\frac{8 \pi G \rho_{\text {tot }}}{3}-\frac{1}{R_{\text {curv }}^{2}} \\
\rho_{\text {tot }} & =\Sigma_{i} \rho_{i}, \quad R_{\text {curv }}^{2} \equiv k / a^{2}
\end{aligned}
$$

where $H$ is the expansion rate, $a(t)$ is the cosmic scale factor (which describes the separation of galaxies during the expansion), $\rho_{\text {tot }}$ is the mass-energy density, and $R_{\text {curv }}$ is the curvature radius. The well known cosmological redshift $z$ (which relates the observed wavelength of a photon $\lambda_{R}$ when received at time $t_{R}$, to its restframe wavelength $\lambda_{E}$ when emitted at time $\left.t_{E}\right)$ is directly tied to the change in cosmic scale factor $a(t): 1+z \equiv \lambda_{R} / \lambda_{E}=a\left(t_{R}\right) / a\left(t_{E}\right)$.

From the Friedmann equation it follows that the total mass-energy and spatial curvature $k$ are linked:

$$
R_{\text {curv }, 0}=H_{0}^{-1} /\left|\Omega_{0}-1\right|^{1 / 2}
$$


where the subscript ' 0 ' denotes the current value of the parameter, $\Omega_{0} \equiv \rho_{\text {tot }} / \rho_{\text {crit }}$ and $\rho_{\text {crit }} \equiv 3 H_{0}^{2} / 8 \pi G$ is the so-called "critical density" that separates positively curved $(k>0)$, high-density universes from negatively curved $(k<0)$, low-density universes. Recent measurements of the anisotropy of the cosmic microwave background have provided convincing evidence that the spatial geometry is very close to being uncurved (flat, $k=0$ ), with $\Omega_{0}=1.0 \pm 0.03$ (deBernardis et al., 2002).

The currently known components of the Universe include ordinary matter or baryons $\left(\Omega_{B}=\rho_{B} / \rho_{\text {crit }}\right)$, cold dark matter $\left(\Omega_{\mathrm{CDM}}\right)$, massive neutrinos $\left(\Omega_{\nu}\right)$, the cosmic microwave background and other forms of radiation $\left(\Omega_{\mathrm{rad}}\right)$, and dark energy $\left(\Omega_{\mathrm{X}}\right)$. The values for these densities are derived empirically, as discussed below, and sum, to within their margins of error, to the critical density, $\Omega_{0}=1$, consistent with the determination of the curvature, $k=0$.

The second set of parameters, which broadly characterize the individual deviations from homogeneity, describe $(\mathrm{a})$ the tiny $(\sim 0.01 \%)$ primeval fluctuations in the matter density as encoded in the CMB, (b) the inhomogeneity in the distribution of matter today, and (c) the possible spectrum of gravitational waves produced by inflation. The initial spectrum of density fluctuations is described in terms of its power spectrum $P(k)$, which is the square of the Fourier transform of the density field, $P(k) \equiv\left|\delta_{k}\right|^{2}$, where the wavenumber $k$ is related to the wavelength of the fluctuation, $k=2 \pi / \lambda$. (Galaxies like ours are formed from perturbations of wavelength $\lambda \sim 1 \mathrm{Mpc}$.) The primordial power spectrum is described by a power law, $P(k) \propto k^{n}$, where a power index $n=1.0$ corresponds to fluctuations in the gravitational potential that are the same on all scales $\lambda$ (so-called scale invariant). The scale-invariant spectrum is predicted by inflation and agrees well with current observations. The overall amplitude of the density perturbations can be described by either $\sqrt{S}$, the CMB quadrupole anisotropy produced by the fluctuations or $\sigma_{8}$, the amplitude of fluctuations on a scale of $8 h^{-1} \mathrm{Mpc}$, which is found from observations to be of order unity.

Accurately measuring these parameters presents a significant challenge. As we now describe, thanks to major advances in technology, the challenge is being met, and in some cases, with independent measurements that check the consistency of both the theoretical framework, and the results themselves. 


\section{B. The Expansion of the Universe}

The expansion of the universe was discovered in 1929 by Edwin Hubble, who measured the distances to a sample of nearby galaxies, and established a correlation between distance and recession velocity. The slope of this relation is the Hubble constant. Large systematic uncertainties in determining distance have made an accurate determination of the Hubble constant a challenge, and only recently have improvements in instrumentation, the launch of the Hubble Space Telescope (HST), and the development of several different measurement methods led to a convergence on its value. Accurate distances to nearby galaxies obtained as part of an HST Key Project have allowed calibration of 5 different methods for determining the distances to galaxies out to $500 \mathrm{Mpc}$ (Freedman et al., 2001). All the techniques show good agreement to within their respective uncertainties, and yield a value

$$
H_{0}=72 \pm 2 \pm 7 \mathrm{~km} \mathrm{sec}^{-1} \mathrm{Mpc}^{-1}
$$

where the error bars represent 1- $\sigma$ statistical and systematic uncertainties, respectively (see Figure 3). Because of the importance of its value to so many cosmological quantities, and because of its historically large uncertainty, $H_{0}$ is often written as $H_{0}=100 h \mathrm{~km} \mathrm{sec}^{-1} \mathrm{Mpc}^{-1}$, so that $h=0.72 \pm 0.02 \pm 0.07$.

The largest contributions to these quoted uncertainties result from those due to the metallicity of Cepheids, the distance to the Large Magellanic Cloud (the fiducial nearby galaxy to which all Cepheid distances are measured relative to), and the calibration of the Wide Field Camera on HST. Other groups using similar techniques (Saha et al., 1997) find a lower value of $\mathrm{H}_{0}(\sim 60 \mathrm{~km} / \mathrm{sec} / \mathrm{Mpc})$. The reasons for the difference are many, as described further in Freedman et al., 2001, but overall the determinations are consistent to within the measurement uncertainties. Recent measurements of $\mathrm{H}_{0}$ based on two completely independent techniques, the Sunyaev-Zeldovich method and the measurement of time delays for gravitational lenses (Keeton et al., 2000; Reese et al., 2000), are yielding values of $\mathrm{H}_{0} \sim$ $60 \mathrm{~km} / \mathrm{sec} / \mathrm{Mpc}$ with systematic errors currently still at the 20-30\% level. New results from the WMAP satellite, discussed in the postscript to this article, give $\mathrm{H}_{0}=71 \pm 4$ $\mathrm{km} / \mathrm{sec} / \mathrm{Mpc}$.

Because light from very distance galaxies was emitted long ago, the Hubble diagram also provides a means of probing the expansion at earlier times. For many decades, efforts have been directed toward measuring what was almost universally expected to be a slowing of 
the expansion over time due to the gravity of all the matter. However, observations by two independent groups have found that supernovae at high redshifts are fainter than predicted for a slowing expansion and indicate that the expansion is actually speeding up (see Figure 4) (Perlmutter et al., 1999; Riess et al., 1998). Although systematic effects due to intervening dust or evolution of the supernovae themselves could explain such a dimming of high-redshift supernovae, several tests have failed to turn up any evidence for such effects. Apparently, the universe is now undergoing an acceleration, with the repulsive gravity of some strange energy form - dark energy - at work. There is weak evidence in the supernova data for an earlier $(z>1 / 2)$, decelerating phase (Turner and Riess, 2002). Such a decelerating phase is expected on theoretical grounds (more later), and establishing its existence (or absence!) is an important goal of future supernova observations.

The remarkable fact that the expansion is speeding up, rather than slowing down, can be accounted for within Einstein's theory, as the source of gravity is proportional to $(\rho+3 p)$, where the pressure $p$ and energy density $\rho$ describe the bulk properties of the "substance". (For ordinary or even nonbaryonic dark matter, $p=0$, while for photons and relativistic particles, $p=\rho / 3$.) A substance that is very elastic, i.e., with pressure more negative than one third its energy density, has repulsive gravity in Einstein's theory (more later). Of course, it could well be that the root cause of cosmic acceleration is not new stuff (i.e., dark energy), but involves a deeper understanding of gravity.

The deceleration parameter was introduced to quantify the slowing of the expansion; it is related to the mass-energy content of the Universe:

$$
q_{0} \equiv \frac{-(\ddot{a} / a)_{0}}{H_{0}^{2}}=\frac{\Omega_{0}}{2}+\frac{3}{2} w_{X} \Omega_{X} \simeq-0.67 \pm 0.25
$$

where $w_{X} \equiv p_{X} / \rho_{X}$ characterizes the pressure of the dark-energy component. ( $w_{X}$ need not be constant; for simplicity, we shall assume it is.) In the absence of dark energy, a flat Universe would decelerate by its own self-gravity (i.e., $q_{0}=0.5$ ), whereas dark energy allows for acceleration. The supernova measurements are consistent with $w_{X}=-1$ and $\Omega_{X}=0.7$. Independent confirmation of such a startling result is extremely important. As discussed below, strong indirect evidence for an additional energy component comes from a comparison of the density of matter with measurements of $\Omega_{0}$ from fluctuations in the CMB.

Dark energy, a "mysterious substance" whose pressure is negative and comparable in magntiude to its energy density, apparently accounts for two-thirds of the matter-energy 
budget of the universe and has no clear explanation. Understanding its nature presents one of the greatest challenges in both cosmology and particle physics.

\section{The Matter Composition of the Universe}

While we know more about the other one-third of the universe - the matter part - important questions remain. According to the current best census, the visible part of ordinary matter - that associated with stars - contributes only about $1 \%$ of the total. What we can see with telescopes is literally the tip of an enormous iceberg.

The rest of the matter in the universe is dark, and its existence is inferred from its gravitational effects. While the case for dark matter holding together galaxies (as well as clusters of galaxies) has been around for a long time (Rubin et al., 1980; Zwickv, 1933), the nature of the dark matter in the universe is still unknown. In fact, we still speak with more certainty about what dark matter is known not to be. Based upon simple accounting, we have all but eliminated the possibility of dark matter being made of neutrons and protons, and established a strong case for a new form of matter.

The accounting of ordinary matter involves three different methods, all of which arrive at the same answer. The most precise of these methods comes from consideration of the formation of light elements during big-bang nucleosynthesis (BBN). Hydrogen, helium, deuterium, and lithium are produced in the first few minutes of the big bang. However, only if the density of ordinary baryons is within a narrow range is the predicted production consistent with what we actually measure (see Figure 5). The production of deuterium is the most sensitive indicator of the baryon density. Measurements made with the 10-meter Keck Telescopes of the amount of deuterium in high-redshift clouds of gas (seen by their absorption of light from even more distant quasars in the Lyman series of lines), together with the theory of big-bang nucleosynthesis yield a density of ordinary matter of $3.8 \pm 0.2 \times 10^{-31} \mathrm{~g} \mathrm{~cm}^{-3}$, or only about $4 \%$ of the critical density (Burles et al., 2001).

Two other determinations are consistent with the nucleosynthesis argument: First, the net absorption of light emitted from very distant quasars by intervening gas (which exists in clouds of gas known as the Lyman-alpha forest after the multiplicity of redshifted absorption features produced by the individual clouds) indicates a similar value for the baryon density. This probes ordinary matter at a time and place when the bulk of the baryons are 
still expected to still be in gaseous form $(z \sim 3-4)$. The second constraint comes from measurements of the CMB, which yield an independent baryon density consistent with that determined from nucleosynthesis. Our best accounting of ordinary matter comes from this early, simpler time, before many stars had yet formed.

Our accounting of baryons at the present epoch, in the local universe, is not as complete. Baryons in stars account for only about one-quarter of all the baryons; the rest are optically dark. While a number of possibilities for the baryonic dark matter (from planets to black holes) have been considered, it now appears that the most plausible reservoir for most of the unseen baryons is warm and hot ionized gas surrounding galaxies within groups and clusters. In fact, in rich clusters the amount of matter in hot intercluster gas exceeds that in stars by a large factor. But since only a few percent of galaxies are found in these unusually rich clusters, the bulk of the dark baryons are still unaccounted for.

While not all of the dark baryons are accounted for, baryonic dark matter itself only accounts for about one-tenth of all dark matter. The evidence that the total of amount of dark matter is much greater - about one-third of the critical density - has gradually become firm, as several, independent (and increasingly higher precision) measures have yielded concordant results (Griest and Kamionkowski, 2000; Sadoulet, 1999).

Clusters of galaxies provide a laboratory for studying and measuring dark matter in a variety of ways. Perhaps most graphically, dark matter can be seen in its effect on more distant background galaxies whose images can be distorted and multiplied by dark-matter gravitational lensing effects. This and other techniques (applied in the x-ray, radio, and optical) have determined the ratio of the total cluster mass to ordinary matter (predominantly in the hot x-ray emitting intracluster gas): averaged over more than fifty clusters the ratio is about 8 (Grego et al., 2001; Mohr et al., 1999). Assuming that clusters provide a "representative sample" of matter in the Universe, the total amount of matter can be inferred from the baryon density. That number is about one third of the critical density.

What then is this nonbaryonic dark matter? The working hypothesis is weakly interacting elementary particles produced in the early universe. Before discussing specific particle candidates, let's review the constraints from astrophysical observations. First, because dark matter is diffusely distributed in extended halos around individual galaxies or in a sea through which cluster galaxies move, dark-matter particles must not interact with ordinary matter very much, if at all. Otherwise, dark matter would by now have dissipated energy 
and relaxed to the more concentrated structures where only baryons are found. At the very least, we can be confident that the constituents of nonbaryonic dark matter are uncharged, and have only very weak interactions.

In addition, the formation of structure in the universe tells us that early on dark-matter particles must have been cold (i.e., moving at non-relativistic speeds) rather than hot (i.e., moving relativistically). If the dark matter had been hot, then these fast-moving particles would have smoothed out the smaller density irregularities, which seed the formation of galaxies and clusters, by streaming from high-density regions to low-density regions. The first objects to form would have been the largest structures (the superclusters) and smaller objects (galaxies) would have only formed later by fragmentation. However, this is inconsistent with observations.

The deep image of the sky obtained by HST in 1995 (the Hubble Deep Field; see Figure 6), along with other observations by ground-based telescopes, identified the epoch when most galaxies formed as a few billion years after the big bang (at redshifts of order 1 to 3). The Sloan Digital Sky Survey, as well as x-ray observations from space and other groundbased telescopes, have shown that clusters form later (redshifts less than about 1). Finally, superclusters, which are loosely bound collections of a few clusters, are forming just today. This sequence is inconsistent with hot dark matter.

Nonetheless, there is at least one hot dark-matter particle that we do know exists - the neutrino. Two experiments, one undertaken in Canada, the other in Japan, now provide

evidence that neutrinos have mass (Ahmad et al., 2002, 2001; Fukuda et al., 2002). The experiments, which are studying solar neutrinos and atmospheric neutrinos, have placed a lower limit on the mass of the heaviest neutrino at about $0.05 \mathrm{eV}$. This implies that neutrinos contribute at least $0.1 \%$ of the mass-energy budget of the universe. However, the cosmological considerations just discussed cap the contribution of neutrinos - or any hot dark matter candidate - to be less than about $5 \%$. This leaves the bulk of the dark matter still to be identified. We will return to the other particle candidates for dark matter later.

\section{The Cosmic Microwave Background}

Today, CMB photons, while very numerous (there are about 2 billion photons for every hydrogen atom) account for a negligible fraction of the mass-energy budget (about $0.01 \%$ ). 
Still, they play a central role in cosmology. First, at early times, the CMB was the dominant part of the mass-energy budget, from which we ascertain that the infant Universe was a hot thermal bath of elementary particles. Second, photons from the CMB interacted closely with matter until the temperature of the Universe had cooled enough for the ionized plasma to combine and form neutral atoms, allowing the photons to stream past. At this "lastscattering surface" of the CMB, the Universe was about 400,000 years old, and about 1100 times smaller than it is today. The CMB is a "snapshot" of the Universe at a much simpler time.

The CMB measurements are a striking example of a new level of precision now being made in cosmology. NASA's COBE satellite, a four-year mission launched in 1989, measured the temperature of the background radiation to better than one part in a thousand, $T_{0}=$ $2.725 \pm 0.001 \mathrm{~K}$ (Mather et al., 1999), and discovered tiny (tens of microKelvin) variations in the temperature of the CMB across the sky. These tiny fluctuations arise from primeval lumpiness in the distribution of matter. In the early Universe, outward pressure from the CMB photons, acting counter to the inward force of gravity due to matter, set up oscillations whose frequencies are now seen imprinted in the CMB fluctuations. Evidence of these "acoustic oscillations" can be seen when the fluctuations are described by their sphericalharmonic power spectrum (see Figures 7-9). In late 2002, the DASI Colloboration detected the last feature predicted for the CMB: polarization (Kovac et al., 2002). Because the CMB radiation is not isotropic (as evidenced by the anisotropy seen across the microwave sky) and Thomson scattering off electrons is not isotropic, CMB anisotropy should develop about a $5 \%$ polarization.

The precise shape of the angular power spectrum of anistropy and polarization depends in varying degrees upon all the cosmological parameters in Table I, and so CMB anisotropy encodes a wealth of information about the Universe. With a host of ground-based and balloon-borne CMB experiments following COBE, a NASA space mission (the Microwave Anisotropy Probe, MAP) now taking new data, and with an European Space Agency (ESA) mission planned for launch in 2007, we are in the midst of realizing the potential of the CMB as a probe of cosmological parameters. A summary of the progress includes determination of the curvature, $\Omega_{0}=1.03 \pm 0.03$, the power law index of density perturbations, $n=$ $1.05 \pm 0.09$, the baryon density $\rho_{B}=4.0 \pm 0.6 \times 10^{-31} \mathrm{~g} \mathrm{~cm}^{-3}$, and the matter density $\rho_{M}=2.7 \pm 0.4 \times 10^{-30} \mathrm{~g} \mathrm{~cm}^{-3}$. The uncertainties in all of these quantities are expected to 
diminish by at least a factor of ten.

As mentioned above, the CMB value for the baryon density is consistent with that determined from BBN. This not only provides confidence that ordinary matter accounts for a small fraction of the total amount of matter, but also is a remarkable consistency test of the entire framework. The CMB provides independent, corroborating evidence for a significant component of dark energy through the discrepancy between the total amount of matter and energy (critical density) and that in matter (1/3 of the critical density). Finally, the measurements of the CMB multipole spectrum are consistent with the emerging new cosmology: a flat Universe with dark matter and dark energy.

Establishing a reliable accounting of the matter and energy in the Universe (see Figure 10) is a major achievement; but, we still have much more to learn about each component and almost everything to understand about the "strange recipe." Moreover, because the energy density of matter, photons and dark energy each change in distinctive ways as the universe expands, the mix we see today must have been different in the past and will be different in the future.

The energy per photon (or per relativistic particle) is redshifted by the expansion (decreasing as $a^{-1}$ ) and the number density of photons is diluted by the increase in volume (as

$a^{-3}$ ), resulting in a total decrease in the energy density proportional to $a^{-4}$. The energy density in matter is diluted by the volume increase of the universe, so that it decreases as $a^{-3}$. The energy density in dark energy changes little (or not at all) as the universe evolves. This means that the Universe began with photons (and other forms of radiation) dominating the energy density at early times $\left(t<10^{4} \mathrm{yrs}\right)$, followed by an era where matter dominated the energy density, culminating in the present accelerating epoch characterized by a transition to a universe dominated by dark energy.

\section{E. The Structure of the Universe Today}

The distribution of galaxies in the local universe reveals a striking, hierarchical pattern with a variety of forms such as galaxy clusters and superclusters, voids and bubbles, sheets and filaments (see Figure 1). In the past 20 years, the volume of the universe surveyed has grown immensely, particularly with the recent development of multi-fiber and multi-slit spectrographs, which allow redshifts to be measured for hundreds of galaxies at one time. In 
the mid-1980's, redshifts for about 30,000 galaxies were measured individually with velocities of up to 15,000 km/sec as part of the CfA survey (Geller and Huchra, 1989).

Unexpected, large-scale structures (walls and bubbles) were revealed with sizes that continued to grow as the survey volumes expanded. In the mid-1990's, about 26,000 additional galaxy redshifts with velocities up to $60,000 \mathrm{~km} / \mathrm{sec}$ were measured with a multi-fiber spectrograph as part of the Las Campanas survey. The larger (but more sparsely sampled) Las Campanas survey found no new larger structures: the universe had finally revealed its homogeneous nature on the largest scales, as expected from the uniformity of the CMB. The most ambitious large-scale-structure surveys to date are the Anglo-Australian Two-degree Field Galaxy Redshift Survey (2dFGRS), which has compiled almost 250,000 redshifts covering about $5 \%$ of the sky, and the on-going Sloan Digital Sky Survey (SDSS), which now has close to half of the 600,000 galaxy redshifts it plans to obtain over about $25 \%$ of the sky.

The simplest description of galaxy clustering is the two-point correlation function, which measures the excess probability over random of finding two galaxies separated by a given distance. It is found empirically to follow a simple power law,

$$
\xi=\left(r / 6 h^{-1} \mathrm{Mpc}\right)^{-1.8}
$$

which implies that finding another galaxy within $6 h^{-1} \mathrm{Mpc}$ from a given galaxy is twice as likely as finding a galaxy within a circle of radius $6 \mathrm{Mpc}$ placed randomly on the sky. The Fourier transform of the correlation function is the previously discussed power spectrum of the distribution of galaxies. The power spectrum can be directly compared with theoretical predictions from inflation and cold dark matter. A complication in this comparison is the extent to which the light observed in galaxies faithfully traces the distribution of mass. It is now known that galaxies are slightly (10\% or so) more clustered than the mass, and that this "biasing" is most pronounced on small scales. That being said, the observed and predicted power spectra (shown in Figure 11) compare well.

On the largest scales, the power spectrum, which measures the level of inhomogeneity today, can also be compared with measurements of the anisotropy of the CMB. This measures the level of inhomogeneity when the Universe was only 400,000 years old and the structure existed only as the seed fluctuations. Because the growth of inhomogeneities depends upon the composition of the Universe, the comparison with theory depends also upon cosmological parameters. When the comparison is made, there is reassuring consistency. 


\section{F. The Age of the Universe}

The time back to the big bang depends upon $H_{0}$ and the expansion history, which itself depends upon the composition:

$$
t_{0}=\int_{0}^{\infty} \frac{d z}{(1+z) H(z)}=H_{0}^{-1} \int_{0}^{\infty} \frac{d z}{(1+z)\left[\Omega_{M}(1+z)^{3}+\Omega_{X}(1+z)^{3\left(1+w_{X}\right)}\right]^{1 / 2}},
$$

where $\Omega_{M}=\Omega_{\mathrm{CDM}}+\Omega_{B}+\Omega_{\nu}$ is the total mass density.

For a universe with a Hubble constant of $72 \mathrm{~km} \mathrm{sec}^{-1} \mathrm{Mpc}^{-1}$ and matter contributing 1/3 and dark energy $2 / 3$ to the overall mass-energy density, the time back to the big bang is 13 Gyr. Taking account of the uncertainties in $H_{0}$ and the composition, the uncertainty in the age of the universe is estimated to be about $\pm 1.5 \mathrm{Gyr}$. The expansion age can also be determined from CMB anisotropy, but without recourse to $H_{0}$, and it gives a consistent age, $t_{0}=14 \pm 0.5$ Gyr (Knox et al., 2001).

The expansion age can also be checked for consistency against other cosmic clocks. For example, the best estimates of the age of the oldest stars in the universe are obtained from systems of $10^{5}$ or so stars known as globular clusters. Stars spend most of their lifetimes undergoing nuclear burning of hydrogen into helium in their central cores. Detailed computer models of stellar evolution matched to observations of globularcluster stars yield ages of about 12.5 billion years, with an uncertainty of about $1.5 \mathrm{Gyr}$ (Krauss and Chabover, 2002). These estimates are also in good agreement with other methods that independently measure the rates of cooling of the oldest white dwarf stars, and techniques that use various radioactive elements as cosmic chronometers (Oswald et al., 1996). Finally, with the assumption that $w_{X}=-1$, the type Ia supernova data can constrain the product of the age and Hubble constant independent of either quantity, $H_{0} t_{0}=0.96 \pm 0.04$ (Tonry et al., 2003). This is consistent with the product of the two, $\left(H_{0}=72 \pm 8 \mathrm{~km} \mathrm{sec}^{-1} \mathrm{Mpc}^{-1}\right) \times\left(t_{0}=13 \pm 1.5 \mathrm{Gyr}\right)=0.96 \pm 0.16$.

In summary, all the ages for the universe are consistent with a consensus age of about $13 \pm 1.5$ Gyr.

\section{G. Recap: The New Emerging Cosmology}

Using a host of diverse observations made possible by advances in technology, we have now defined the basic features of the Universe: it is spatially flat and 13 Gyr old with a 
currently accelerating expansion, comprised of one third dark matter and two thirds dark energy. The variety and redundancy of observations is important: the overall picture does not depend on just a single measurement or type of measurement. Consistency checks on the age, the baryon and matter densities, and the total density and spatial curvature have increased our confidence both in the description as well as in the overall framework. We now move from discussion of the basic features of the universe to the underlying physics that forms the basis for our current understanding.

\section{UNDERSTANDING THE UNIVERSE}

In addition to breakthrough observations, creative new ideas are also driving progress in cosmology. Not only do we know more about the universe, but our understanding is deeper, and the questions that we are asking are more profound. Still, our understanding of the origin and evolution of the universe has not yet caught up with what we know about it. But a vital ingredient in furthering our understanding and shaping our questions has been the recognition of the connections that exist between the elementary particles on the smallest scales, and the universe on the largest.

\section{A. Origin of Structure}

The abundance of structure that has been mapped out today - from galaxies of mass as small as $10^{6} M_{\odot}$ to superclusters of mass exceeding $10^{16} M_{\odot}$ - speaks to a remarkable transformation that occurred in the early universe as small primeval inhomogeneities in the

distribution of matter were amplified by the attractive forces of gravity $\left(1 M_{\odot} \simeq 2 \times 10^{33} \mathrm{~g}\right.$ refers to the mass of the sun).

During the earliest moments the primordial fluctuations did not grow because the expansion was controlled by radiation, and radiation does not clump. When the universe became matter-dominated, the inhomogeneity then grew at precisely the same rate as the cosmic scale factor, $(\delta \rho / \rho) \propto a(t)$. Because the size of the universe grew by a factor of about 10,000 during the matter dominated era, initial fluctuations of amplitude $0.01 \%$ or so are all that is needed to seed the nonlinear structure seen today. Formation of structure then ceased a few billion years ago when the accelerated expansion began, pushing the existing 
structures apart.

The required primordial lumpiness should have left its signature on the CMB in the form of temperature fluctuations of order ten microKelvins. And this is precisely what has been measured, both in amplitude and variation with angular scale (cf. Figure 9) . Being able to account for how the structure seen today arose from a nearly homogeneous beginning is a major success of the FLRW model.

Of course, a natural question arises: What is the origin of the primeval lumpiness? Cosmologists have a working model for this: the seeds of structure arose from the stretching and conversion of quantum noise originating on subatomic length scales to density inhomogeneity on astrophysical length scales, caused by a tremendous burst of expansion called inflation. Inflation, coupled with the idea of including nonbaryonic dark matter in the universe, has led to a predictive and descriptive theory of how the structure in the universe plausibly arose. This descriptive theory is known as Cold Dark Matter (CDM) (Blumenthal et al., 1984). From the simple starting point of cold dark matter and inflation-produced lumpiness follows a highly successful picture for the formation of structure in the universe.

The defining prediction of CDM is that structure forms from the "bottom up": first galaxies, then clusters of galaxies, and finally superclusters. This ordering, now confirmed by observations, follows from the fact that the degree of lumpiness is larger on smaller scales, so that the smaller objects become gravitationally bound, stop expanding, and collapse back on themselves first.

As computing power and numerical techniques have improved, simulations of the evolution of structure in the universe have become increasingly sophisticated, providing additional insight into the evolution of cosmic structure. The initial ingredients for these simulations are specified by the CDM paradigm, and the ensuing growth of structure via the force of gravity is now computed by following the motions of more than a billion particles. The properties of the simulated universe (correlation function, power spectra, and masses and abundances of galaxies, etc) are calculated for different recipes (dark matter and baryon densities, with and without dark energy, and different values of the cosmological parameters) and can all be compared with observations. Numerical simulations were instrumental in demonstrating the failure of structure formation in the presence of hot dark matter, and the success of flat CDM models with dark energy in matching the observed distribution of structure seen today. The successful predictions of CDM go beyond being merely descriptive, 
and now include many quantitative predictions.

That being said, the successes of CDM largely involve predictions that follow from the basic paradigm and the action of gravity alone. However, understanding the development of the structure we see with telescopes requires an understanding of how baryons form into stars in galaxies. Complicated gas dynamics come into play and there may even be feedback from the energetics of star formation on the dark-matter structures themselves. Crisp predictions become more difficult. Understanding how real galaxies form and evolve is a rich subject with many outstanding questions whose answers will require more observations as well as detailed astrophysical modelling that goes beyond the gravity of cold dark matter alone. Pinning down the basic cosmological framework has helped significantly by removing uncertainty associated with the evolution of the Universe.

\section{B. The Expansion}

Hubble's discovery of the expansion of the universe changed our cosmic perspective forever - we now know that we live in an evolving universe with a big-bang beginning. Its interpretation within Einstein's theory of gravity was a striking confirmation of the dynamical nature of space and time - the expansion is due to the stretching of space itself. However, within the framework of general relativity there is no answer to the most basic question what happened just before the big bang to get the expansion going? According to general relativity, the big bang was the singular creation of matter, energy, space and time itself. If correct, then there was no "before" before the big bang - a neat and tidy, if not entirely satisfying, answer.

Since Einstein's theory does not incorporate quantum mechanics, there is reason to believe that it is not complete or fully applicable around the time of the big bang. Answering the "before the big bang" question most likely still depends upon marrying quantum mechanics to gravity, and applying that merged theory to the earliest moments of the universe. Currently, superstring theory is the most promising idea for making such a connection, and we will return later to some of the speculations based upon it.

Even within general relativity there are conceptual questions about the beginning. Not all big-bang models necessarily lead to a universe like ours. Unless the initial conditions were "just so" (see, e.g., Hogan, 2000), the universe might well have recollapsed long ago; or it 
might have gone into a coasting phase of indeterminate duration; or the expansion need not necessarily have been the same in all directions (isotropic), or there might not necessarily have been large-scale regularity in the distribution of matter (homogeneity), as we observe in the universe today.

Apparently a special beginning is required. There are two ways to read this: The first is that the state of the universe today accurately pins down the initial conditions, a point of view advocated by a few (Penrose, 1979 ). The second is to look for a way to get around the special conditions, a road that led to the idea of cosmic inflation.

In 1980, Alan Guth pointed out that an early, brief period of very rapid (indeed exponential) expansion, now called inflation, could change the cosmic landscape dramatically (Guth, 1980). He had in mind a scenario wherein the universe got trapped in a "false-vacuum state" during a cosmological phase transition associated with the breaking of symmetry between the strong, weak and electromagnetic forces. Although this specific idea does not seem to work, it nevertheless led to a paradigm for inflation based on the potential energy associated with an as-of-yet hypothetical scalar field called the inflaton (AlbrechtSteinhardt, 1982; Linde, 1983). (If it exists, this field is distantly related to the Higgs scalar field that particle physicists believe explains why particles have mass.)

According to inflation, small bits of the universe underwent a burst of expansion when the universe was extremely young $\left(t \ll 10^{-2} \mathrm{sec}\right)$. This expansion flattened the local geometry in the same way inflating a balloon makes a fixed region look flatter and smoother as the balloon inflates (in the case of the universe the blow-up factor exceeded a factor of $10^{40}$ !). The conversion of the scalar-field potential energy into particles and photons accounts for the tremendous heat content of the big bang and initiates the early, radiation-dominated era. During the conversion of scalar field energy, quantum fluctuations in the inflaton field on subatomic scales, stretched to astrophysical size by the tremendous expansion, became the seed density inhomogeneities. Moreover, quantum fluctuations in the metric of spacetime itself lead to a predicted spectrum of long-wavelength gravitational waves.

Thus, cosmic inflation explains the smoothness of the universe, the heat of the big bang, and it predicts a flat universe with characteristic seed irregularities in the matter distribution and a spectrum of gravity waves. It also says that all we can see arose from "our big bang," one of many bursts of inflationary expansion that took place early on.

As of yet there is no single, agreed-upon model of inflation. However, all models are 
based upon the paradigm described above and make three firm predictions: (i) a spatially flat universe; (ii) a nearly scale-invariant distribution of density perturbations; and (iii) a nearly scale-invariant spectrum of gravitational waves.

In the early 1980s when inflation was gaining sway with cosmological theorists, its prediction of a flat universe looked to be its downfall. The best measurements indicated that matter contributed only about $10 \%$ of the critical density, although the uncertainties were quite large given that the amount of dark matter was still largely undefined. But as we saw above, recent determinations indicate that the universe is almost certainly flat, with dark matter contributing one third of the critical density and dark energy the other two thirds.

The seed inhomogeneities of inflation were impressed upon the universe very early on. This results in a kind of synchronized motion of irregularities on different length scales, and leads to a characteristic pattern of "acoustic peaks" in the multipole power spectrum of the CMB anisotropy. Measurements now show a clear pattern of acoustic peaks, cf. Figure 9. Their relative heights also indicate that the seed perturbations are consistent with being almost scale-invariant, again as inflation predicts.

Inflation has passed its first round of tests. Over the next decade additional observations, especially those coming from measurements of the CMB, will test inflation more decisively and may shed light on how the inflaton field fits into the larger picture of particle physics.

The spectrum of gravitational waves extending from wavelengths of kilometers to billions of light years are certainly beyond the reach of the current generation of Earth-based gravitywave detectors. There is some hope that gravity-wave-induced polarization signatures in the cosmic microwave background can be detected with a new generation of experiments. If so, not only would the third prediction of inflation be tested, but also the time when inflation took place would be identified.

While inflation is making a good case for being included in the standard cosmological model, it does not address the biggest question, how the Universe began. Superstring theory shows promise of shedding more light on how the Universe began. With superstring's prediction that there are more than three spatial dimensions, it opens new dimensions in cosmology, both figuratively and literally. While there have yet to be successes or even compelling predictions, superstring theory has evoked intriguing cosmological ideas. The "brane world" idea holds that our universe is actually a three-dimensional "sheet" in a much higher dimensional space where gravity exists in the full spacetime, but other forces 
and particles are confined to only three dimensions. From this paradigm has come the idea that the big bang might have been the collision of two such sheets, and even further that such collisions happen repeatedly, adding new life to the old oscillating universe scenario. Such ideas are not yet well developed enough to be testable, but their creativity speaks to the vitality of cosmology. And it should be remembered that about twenty years ago inflation seemed radical and untestable!

\section{The Composition of the universe}

Thirty years ago the universe seemed much simpler. We only had knowledge of ordinary matter, and even the fact that most of the ordinary matter did not reside in stars was still to be discovered. Today, we have a much more complete (and correspondingly much more complicated) accounting, with five components: ordinary matter, massive neutrinos, cold dark matter, dark energy, and photons, cf Figure 10. And now even the "ordinary" matter is not simple - the bulk of it is dark, and in a form yet to be firmly identified.

The leading candidates for the CDM particle are the axion and the neutralino, two hypothetical elementary particles. If they exist, these particles would have been produced in the earliest moments of the universe, and survived in sufficient numbers to account for the dark matter. Both are new forms of stable matter, predicted to exist by theories that attempt to unify the forces of Nature. But they have wildly different masses: a trillion times smaller than that of the electron for the axion, and a hundred times larger than the mass of the proton for the neutralino.

If the cold dark matter hypothesis is correct, then the halo of our own Milky Way should be awash in axions or neutralinos (or some other slowly moving particle). While the interactions of axions and neutralinos with ordinary matter are very weak (and can be neglected in almost all circumstances) specialized detectors have already been built to confirm (or rule out) their presence. In addition, the neutralino, the lightest of a new class of particles predicted by superstring theory, has two other signatures of its existence. It can be produced at a particle accelerator, given sufficient energy. Alternatively, high-energy neutrinos

produced by the annihilation of the few neutralinos that are captured by the Sun could be detected; or positrons and/or gamma rays produced by neutralinos annihilating in the halo might be found. With the efforts underway, evidence that axions or neutralinos comprise 
the cold dark matter could be forthcoming in the next decade (Griest and Kamionkowski, 2000; Sadoulet, 1999).

Our state of understanding of the origin of the various components comprising the universe varies widely. If inflation is correct, then photons arose from the decay of the potential energy of the scalar field that drove inflation. The existence of quark-based matter that we are made of involves three elements: the action of microscopic forces that do not conserve the net number of quarks (baryon-number violation) and break the symmetry between particles and antiparticles (referred to as $\mathrm{CP}$ violation), and a departure from thermal equilibrium. These three conditions, first spelled out by Russian dissident and physicist Andrei Sakharov in 1967, are necessary for the universe to develop a slight excess of baryons over antibaryons. When the universe was around $10^{-5}$ sec old, the bulk of the baryons and antibaryons annihilated, leaving the few residual baryons for every 10 billion photons that now constitute the ordinary matter we see today. The details of "baryogenesis" - which may even involve neutrinos - are not fully understood. Critical in this regard is a better understanding of CP violation and neutrinos.

In some ways the emergence of neutrinos and cold dark matter from the post-inflation thermal bath is better understood. At very early times, when temperatures were extremely high, a kind of particle democracy existed, with roughly equal numbers of all particle types. As the temperature dropped below the point where a given species could be still be produced in pairs (i.e., where the thermal energy $k T$ is less than its rest mass energy $m c^{2}$ ), the numbers of those particles and antiparticles decreased rapidly through mutual annihilation. Because of their small masses and their small annihilation cross sections, neutrinos never annihilate, leaving them as abundant today as CMB photons. Once their masses are known, their contribution to the mass density follows directly. From what we know about neutrino masses, their contribution is at most comparable to that of ordinary matter, too small to account for the bulk of the dark matter. Nonetheless, neutrinos validate the basic idea of dark matter in the form of something other than baryons and do play a small role in the formation of structure.

The neutralino story is more complicated. Cosmic neutralinos do annihilate significantly; however, once their abundance falls to about 1 per billion photons, they become so rarified that they cease annihilating. Their relic abundance, determined by their annihilation cross section, turns out to be in the right range to account for the dark matter. 
Dark energy is the largest and most perplexing component of the universe. The simplest possibility, that it is the energy associated with quantum vacuum fluctuations, suffers from the fact that calculating how much "quantum nothingness weighs" has eluded theorists for more than five decades and naive estimates are at leats 55 orders-of-magnitude too large! This suggests to some that ultimately it will be shown that "even quantum nothingness weighs nothing," because that outcome seems more likely than finding a means to reduce the naive estimate by precisely 55 orders-of-magnitude or more. Yet, if the dark energy is not quantum vacuum energy, what is it? A host of possibilities have been suggested, from a mild version of inflation involving an extremely light scalar field to the influence of new physics occurring in the extra spatial dimensions, as predicted by superstrings. However, the small magnitude of the dark energy is not the only problem. Another is trying to understand why at this point in time, the dark energy is only just beginning to dominate the expansion. It seems an odd coincidence, and one that so far defies explanation.

Finally, what can we say about the destiny of the universe? In the simple universe containing only matter, the destiny of the universe is linked to its geometry: uncurved and negatively curved universes expand forever; and positively curved universes recollapse. While we have determined that we live in an uncurved universe, adding dark energy to the mix severs the link between geometry and destiny. Depending upon the nature of the dark energy, a flat universe can continue accelerating forever (if the dark energy is quantum vacuum energy), or it can slow down or even recollapse if the dark energy dissipates with time (Krauss and Turner, 1999).

\section{LOOKING FORWARD}

In the last two decades, a set of interesting ideas based upon unexpected connections between the quarks and the cosmos and the emergence of a new generation of observations and experiments have transformed cosmology into a full-fledged, precision science. The tenmicroKelvin fluctuations in the cosmic microwave background radiation are constraining cosmological parameters and shedding light on the earliest moments of the universe. Maps of the distribution of galaxies and clusters in volumes approaching billions of megaparsecs on a side are testing the cold dark matter paradigm. The current expansion rate of the universe has finally been pinned down to $10 \%$ precision, and measurements of the past rate 
have revealed we are now in a period of cosmic acceleration. The contribution of ordinary matter to the overall mass-energy budget has been shown to be small, with more than $95 \%$ of the universe existing in new and unidentified forms of matter and energy.

Many, creative theoretical ideas have emerged that provide a way to understand the expansion of the universe, its composition, and the origin of structure. Still, big questions remain. Why are there three different forms of matter/energy of comparable abundance, with the transition to accelerated expansion occurring very recently? How much of the truth does inflation hold about the early universe and what is the hypothetical inflaton field that drove inflation? What is the dark matter and the strange dark energy? Could the complicated recipe and accelerated expansion indicate that we don't yet fully understand gravity?

Astronomers and physicists are in the midst of carrying out ambitious new experiments, completing large surveys of the Universe, and commissioning powerful new telescopes with novel technology and advanced instrumentation. There is great promise of increasingly sharper tests of inflation, cold dark matter, and dark energy, and always the potential for further new surprises. There is no doubt that we are in the midst of a revolutionary period of discovery in cosmology.

\section{WMAP POSTSCRIPT}

Three months after we submitted this Colloquium article, the WMAP Collaboration presented results from their first year of data. ${ }^{1}$ The results were at the same time stunning and unsurprising. As several cosmologists put it, the biggest surprise was the lack of a surprise. Overlapping and precision measurements have elevated cosmology to a new maturity, where consistency is becoming a hallmark.

The angular power spectrum (cf Figure 9) was derived from five all-sky maps with maximum angular resolution of $0.2^{\circ}$ (30 times that of COBE) at frequencies from $20 \mathrm{GHz}$ to 100 $\mathrm{GHz}$ (reference). The measurements were calibrated from the Doppler shift of the CMB arising from Earth's motion around the sun, $\delta T=(v / c) T_{0} \simeq 0.27 \mathrm{mK}\left(v / c=10^{-4}\right)$. WMAP's

\footnotetext{
${ }^{1}$ When the results were announced, cosmologists were pleased to learn that the MAP satellite had been re-named the Wilkinson Microwave Anisotrophy Probe (WMAP) to honor David Wilkinson, a pioneer in the study of the CMB and a leader of the MAP project, who died in September 2002.
} 
location a million miles from Earth helped keep systematics to below $0.5 \%$. From $\ell=2$ to $\ell \sim 350$ the measurements of the multipole amplitudes were limited by sample (or cosmic) variance. (Theories like inflation do not predict values for the individual multipoles, but rather the variance of the distribution from which they are draw. The fact that for a given $\ell$ only $2 \ell+1$ multipoles can be measured limits the precision with which the variance can be estimated.)

The WMAP results (Bennett et al., 2003) have sharpened and put on firmer footing a large number of cosmological parameters (see Table I). The consistency of WMAPdetermined parameters with previous values was a strong indication of the increasing reliability of cosmological results and their error estimates. In particular, WMAP strengthened the case for dark matter by its measurement of the ratio of the total amount of matter to that in baryons, $\Omega_{M} / \Omega_{B}=6 \pm 0.04$, and the case for dark energy by showing that something like a cosmological constant is needed to "balance the books," $\Omega_{X}=0.7 \pm 0.04$. WMAP made clear that our current consensus cosmology rests on a strong and diverse, interlocking set of measurements.

While WMAP has yet to map CMB polarization (though it is in the works), by detecting the cross correlation between polarization and temperature anisotropy it found the signature of the re-ionization from UV starlight of the first stars at a redshift $z \simeq 20 \pm 10$. This is consistent with the predictions of the CDM paradigm, and together with the SDSS quasars with redshifts greater than 6 this now nicely brackets the re-ionization history of the Universe: at $z \sim 20$ the fraction of free elections rose to around $50 \%$ and by $z \simeq 6$ it exceeded $99.99 \%$.

Two months after the WMAP results, a new compilation and analysis of over 200 type Ia supernovae was presented (Tonry et al., 2003), and the direct evidence for cosmic acceleration also grew stronger. In particular, if dark energy is assumed to have $w=-1$ (like a cosmological constant), the supernovae data imply

$$
\begin{aligned}
\Omega_{X}-1.4 \Omega_{M} & =0.35 \pm 0.14 \\
q_{0} & =-0.66 \pm 0.10 \\
\Omega_{X} & =0.77 \pm 0.06 \quad \text { assuming } \Omega_{M}=0.3 \pm 0.04 .
\end{aligned}
$$




\section{References}

Ahmad, Q. R., et al. 2002, Phys. Rev. Lett. 89, 011302.

Ahmad, Q. R., et al. 2001, Phys. Rev. Lett. 87, 071301.

Albrecht, A. and P. J. Steinhardt, 1982, Phys. Rev. Lett. 48, 1220.

Bennett, C. L., et al. 2003, Astrophys. J., in press (astro-ph/0302207).

Blakeslee, J. P., et al. 2002, Mon. Not. Roy. Astron. Soc. 330, 443.

Blumenthal, G. R., S. M. Faber, J. R. Primack, and M. J. Rees, 1984, Nature 311, 517.

Burles, S., et al. 2001, Astrophys. J. 552, L1.

Cowan, J. J., F. -K. Thielemann, and J. W. Truran, 1991, Ann. Rev. Astron. Astrophys. 29, 447.

de Bernardis, P., et al. 2002, Astrophys. J. 564, 559.

Elgaroy, O., et al. (2dF Colaboration) 2002, Phys. Rev. Lett. 89, 061301.

Freedman, W. L., et al. 2001, Astrophys. J. 553, 47.

Fukuda, S., et al. 2002, Phys. Rev. Lett. B539, 179.

Geller, M. and J. Huchra, 1989, Science 246, 857.

Gorski, K. M., et al. 1996, Astrophys. J. Lett. 464, 11.

Grego, L., et al. 2001, Astrophys. J. 552, 2.

Griest, K. and M. Kamionkowski, 2000, Phys. Rep. 333-4, 167.

Guth, A. H., 1980, Phys. Rev. D20, 30.

Hogan, C. J., 2000, Rev. Mod. Phys. 72, 1149.

Keeton, C. R., et al. 2000, Astrophys. J. 542, 74.

Kinney, W., A. Melchiorri, and A. Riotto, 2001, Phys. Rev. D 63, 023505.

Knox, L., N. Christensen, and C. Skordis, 2001, Astrophys. J. 563, L95.

Kovac, J., et al. 2002, astro-ph/0209478.

Krauss, L. M., and B. Chaboyer, 2002, Science, in press, astro-ph/0111597.

Krauss, L. M. and M. S. Turner 1999, Gen. Rel. Grav. 31, 1453.

Lahav, O., et al. 2002, MNRAS 333, 961.

Lewis, A., and S. Bridle, 2002, astro-ph/0205436.

Linde, A. D., 1983, Phys. Lett. 129B, 177.

Mather, J. C., et al. 1999, Astrophys. J. 512, 511.

Mohr, J., B. Mathieson, and A. E. Evrad, 1999, Astrophys. J. 517, 627. 
Oswald, T. D., J. A. Smith, M. A. Wood, and P. Hintzen, 1996, Nature 382, 692.

Penrose, R., 1979, in General Relativity: An Einstein Centenary Survey, edited by S. W. Hawking and W. Israel (Cambridge University Press, Cambridge), p. 581.

Perlmutter, S., et al. 1999, Astrophys. J. 517, 565.

Perlmutter, S., M. S. Turner, and M. White, 1999, Phys. Rev. Lett. 83, 670.

Pryke, C., et al. 2002, Astrophys. J. 568, 46.

Reese, E. D., et al. 2000, Astrophys. J. 533, 38.

Riess, A., et al. 1998, Astron. J. 116, 1009.

Rubin, V., W. K. Ford, and N. Thonnard, 1980, Astrophys. J. 238, 471.

Sadoulet, B., 1999, Rev. Mod. Phys. 71, S197.

Saha, A., et al. 1997, Astrophys. J. 486, 1.

Sandage, A. R, 1970, Physics Today 23, 34 .

Sievers, J. L., et al. 2002, in press, astro-ph/0205387.

Tegmark, M., A. J. S. Hamilton, and Y. Hu, 2002, MNRAS 335, 887.

Tonry, J. L., et al. 2003, Astron. J., in press (astro-ph/0305008).

Turner, M. S., 2002, Astrophys. J. 576, L101.

Turner, M. S. and A. Riess 2002, Astrophys. J. 569, 18.

Zwicky, F., 1933, Helv. Phys. Acta 6, 510. 


\section{Figures}

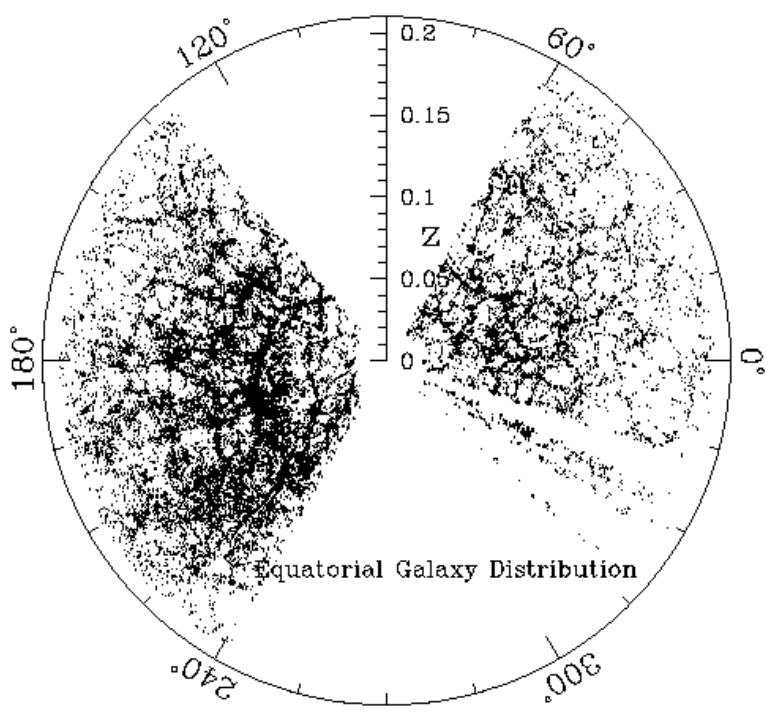

FIG. 1 The Universe observed: A slice of the Universe constructed from the positions of 60,000 galaxies in the Sloan Digital Sky Survey. Voids and walls can be clearly seen (image courtesy of SDSS). 


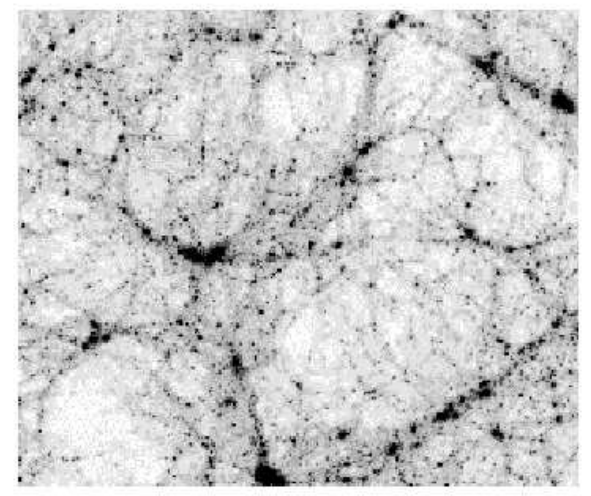

FIG. 2 The Universe simulated: The distribution of dark matter in a large-scale numerical simulation of the Universe. The cosmic web of dark matter - with its sheets, sinuous filaments and voids is apparent (image courtesy of the Virgo Consortium). 


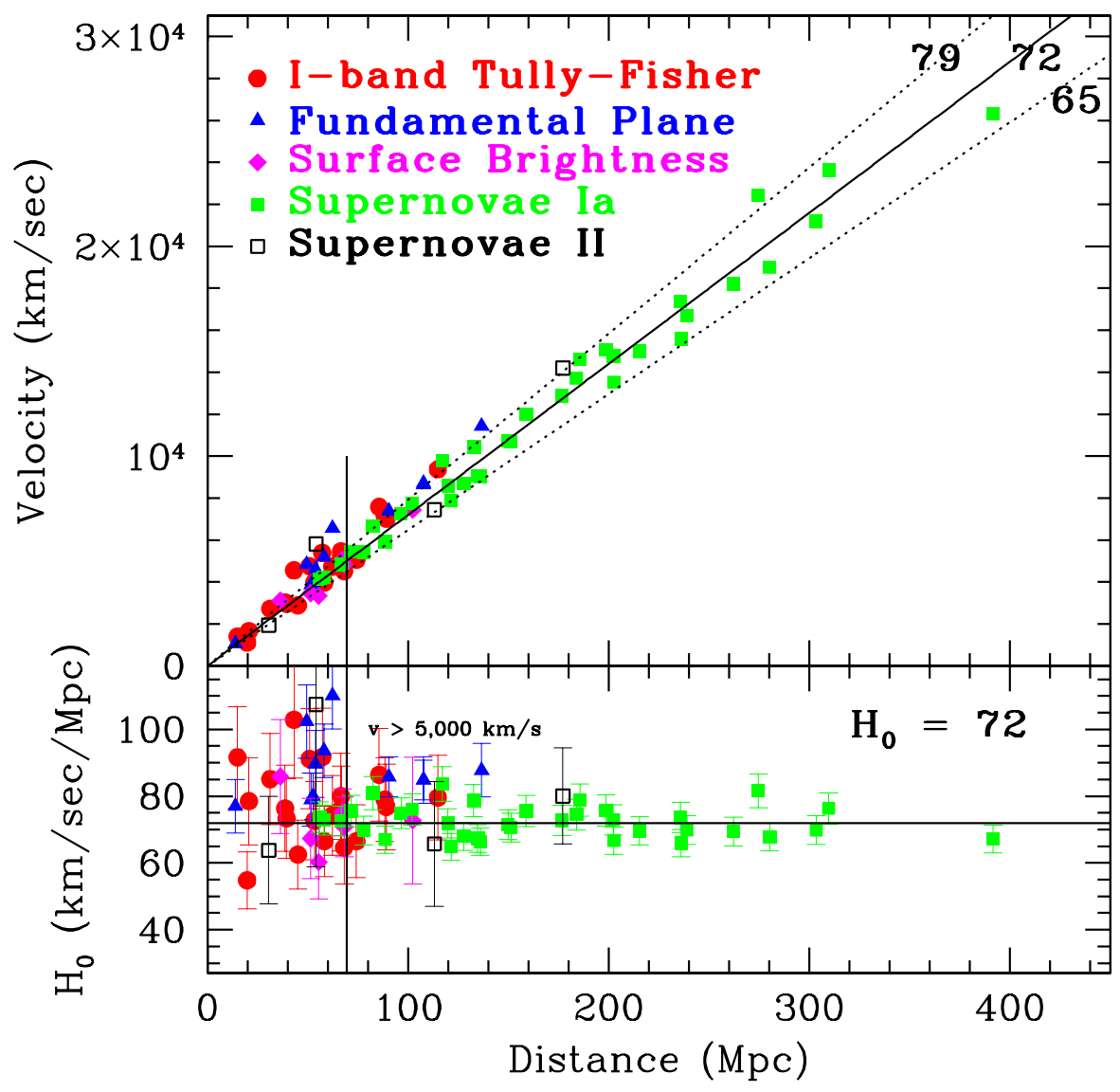

FIG. 3 Hubble diagram: Low-redshift galaxies are used to establish the expansion of the Universe and the Hubble constant; the consistency of the five different distance indicators is shown. The lower panel shows the value of the Hubble constant object by object and the convergence to $72 \mathrm{~km} / \mathrm{s} / \mathrm{Mpc}$. The scatter at distances less than $100 \mathrm{Mpc}$ arises due to gravitational induced "peculiar velocities" that arise from the inhomogeneous distribution of matter. 


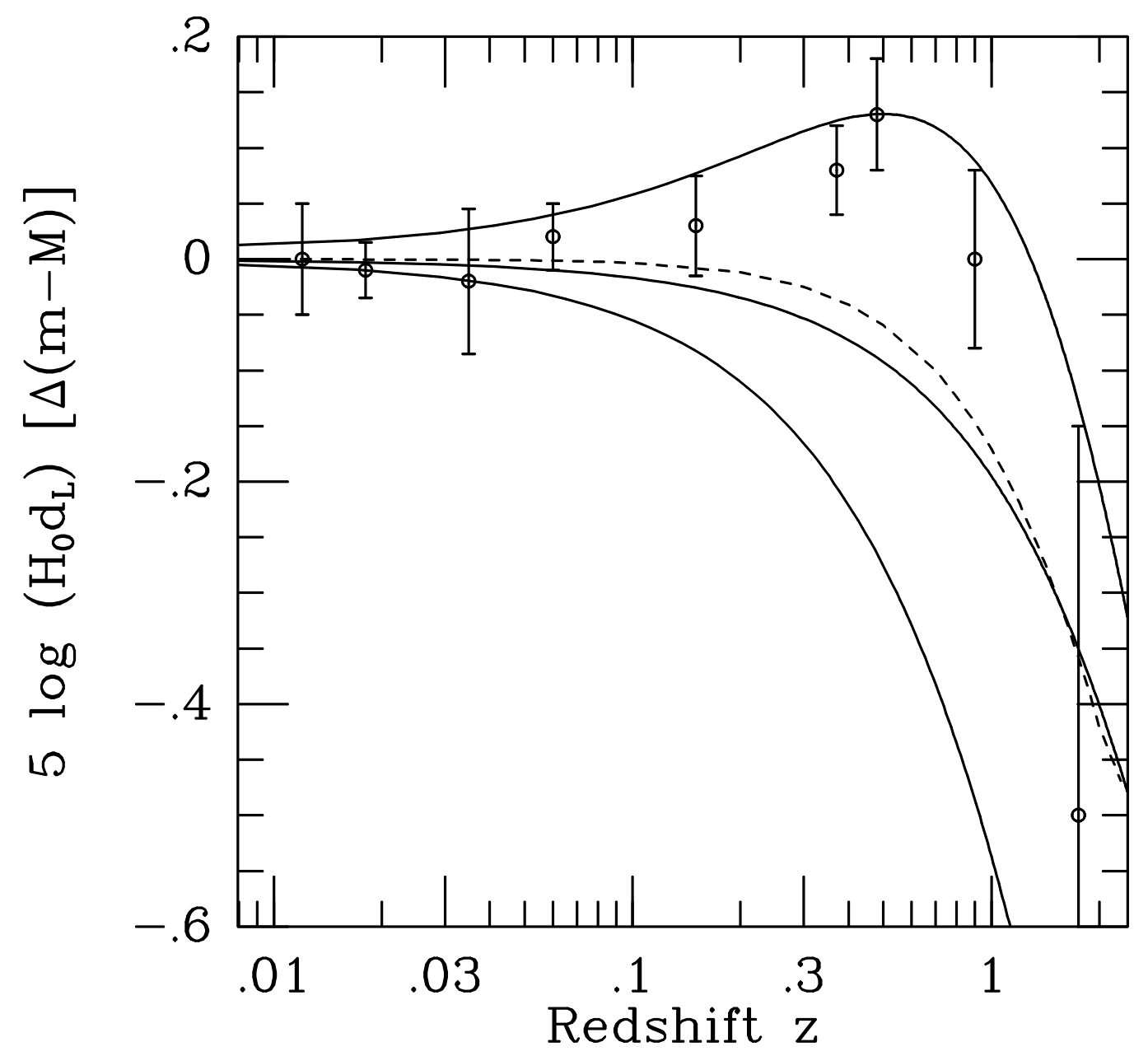

FIG. 4 Hubble diagram: High-redshift type Ia supernovae probe the expansion history and reveal accelerated expansion. In this differential Hubble diagram the distance modulus, which is 5 times the logarithm of the distance, relative to an empty Universe $\left(\Omega_{0}=0\right)$ is plotted. Measurements from more than 200 type Ia supernova are binned into 9 data points. The solid curves represent three theoretical models: from the top, $\Omega_{\Lambda}=0.7$ and $\Omega_{M}=0.3 ; \Omega_{\Lambda}=0$ and $\Omega_{M}=0.3$; and $\Omega_{\Lambda}=0$ and $\Omega_{M}=1$. The broken curve represents a nonaccelerating, flat Universe (i.e., $q=0$ for all $z$ ); points above this curve indicate acceleration (adapted from data in Tonrv et al., 2003). 


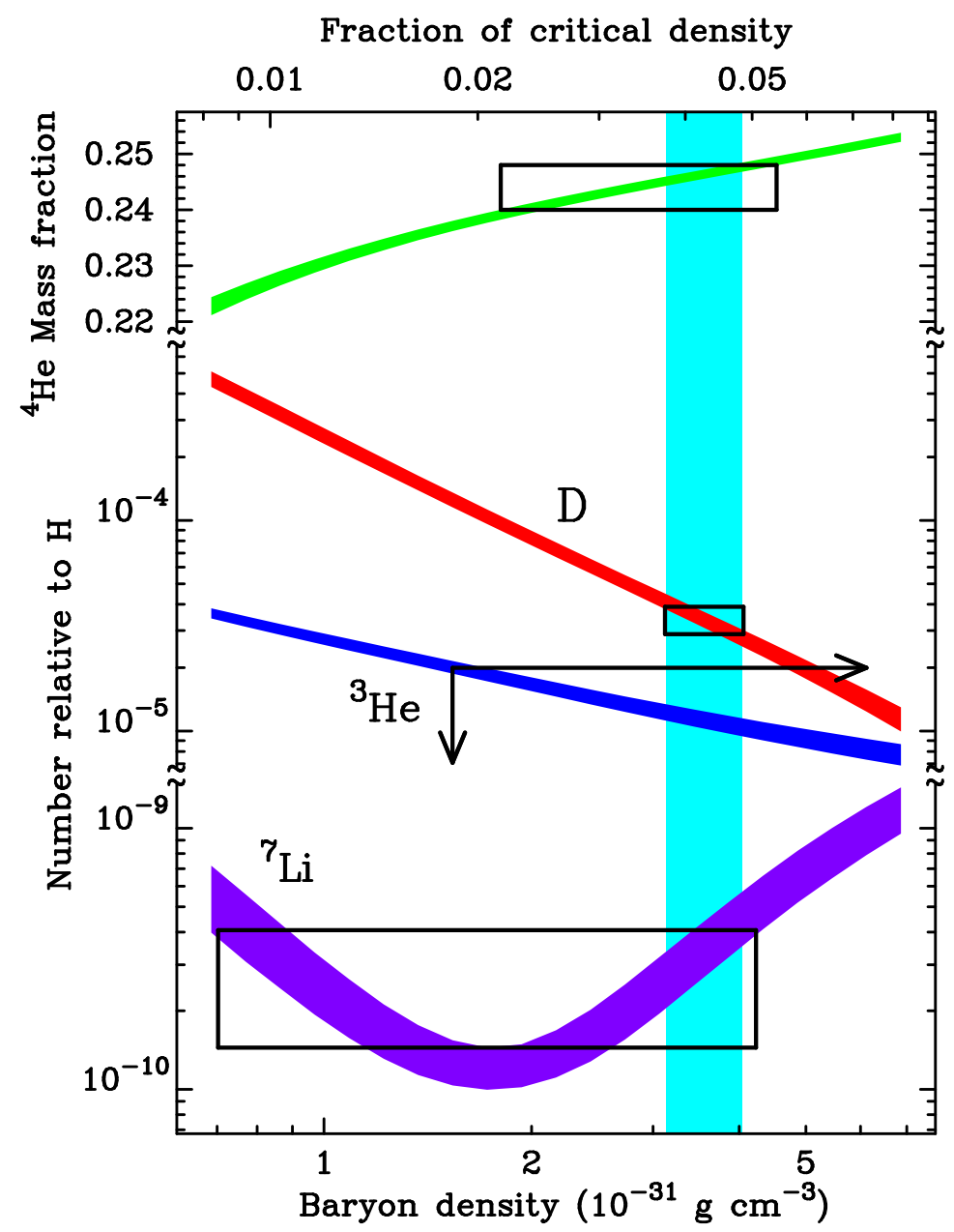

FIG. 5 The predicted abundance of the light elements vs. baryon density. The vertical band indicates the narrow range of baryon densities consistent with the deuterium measurements; the boxes (and open box for ${ }^{3} \mathrm{He}$ ) indicate the range in baryon density (horizontal extent of box) that is consistent with the measured light-element abundance (vertical extent of box). The overlap of the boxes with the deuterium band indicates the general consistency of the observed abundances of the other light elements with their predicted abundances for this baryon density. (Note, for the $\Omega_{B}$ scale at the top, $h^{2}=0.5$ is assumed.) 


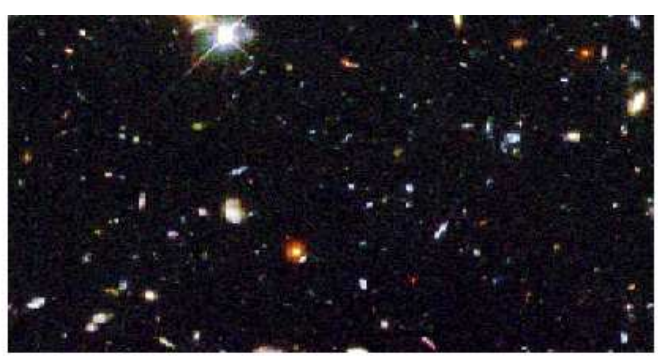

FIG. 6 The deepest image of the sky in visible light obtained by the Hubble Space Telescope in 1995 (the Hubble Deep Field). This image revealed the time when typical galaxies (like our own Milky Way) were forming (redshifts $z \sim 1-3$ ). In this image of about one-forty-millionth of the sky, there is one star and more than 1500 galaxies (image courtesy of NASA). 

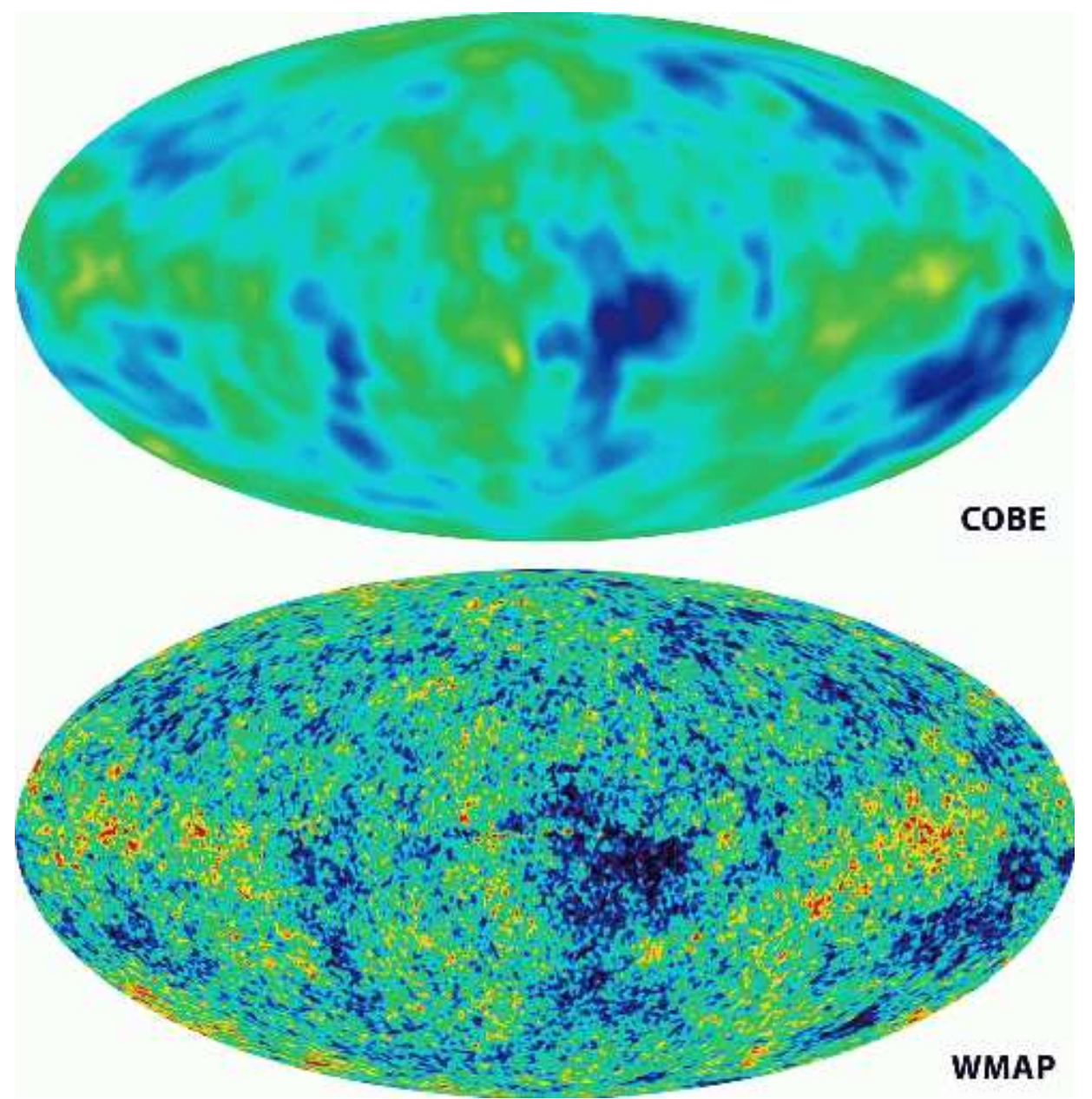

FIG. 7 Anisotropy of the Cosmic Microwave Background: All-sky maps, made by COBE (upper) and by WMAP (lower); range of color scale is $\pm 200 \mu$ Kelvin. The consistency of the 30 times higher resolution and higher sensitivity WMAP results with COBE is apparent (courtesy of NASA/WMAP Science Team). 


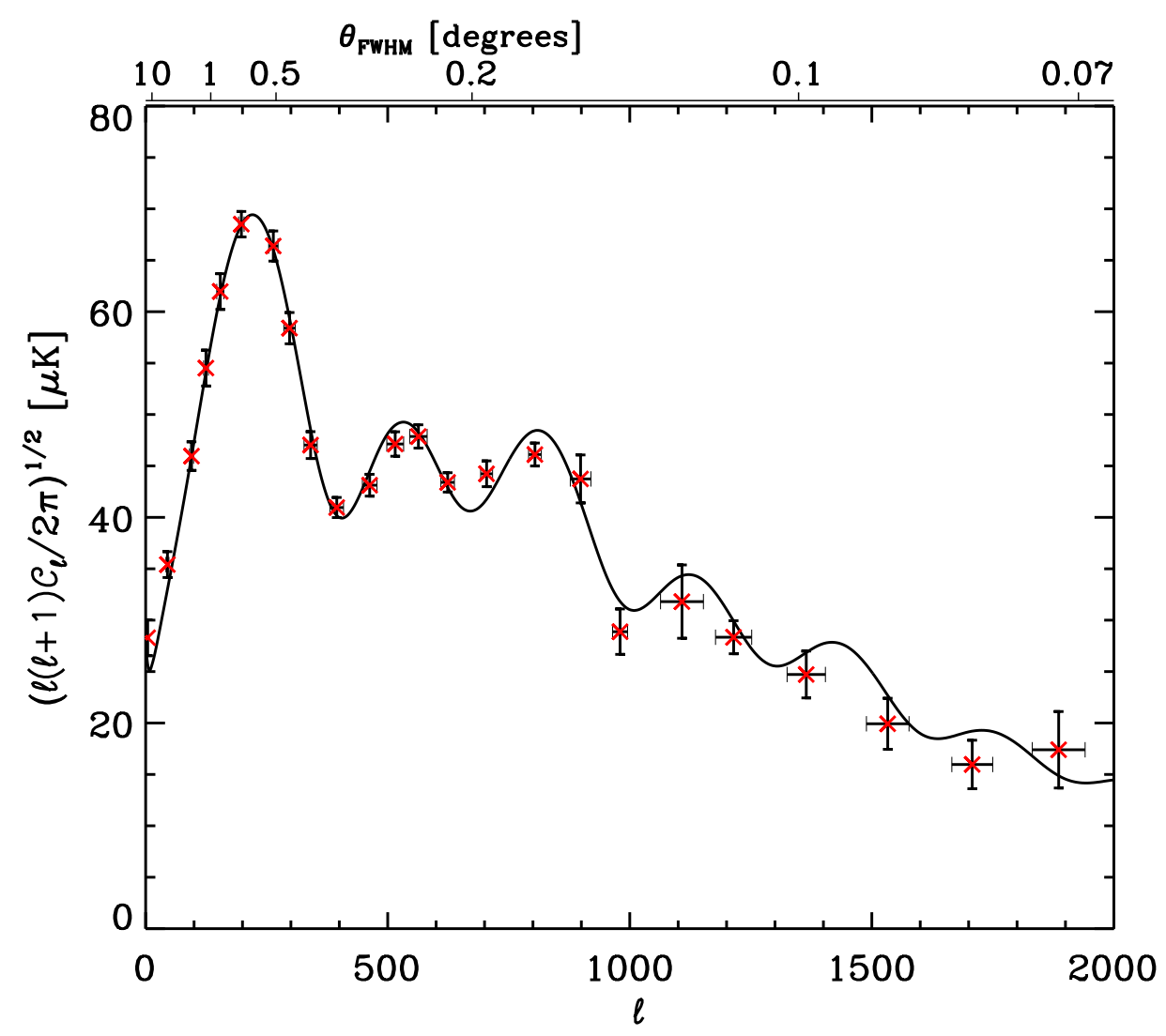

FIG. 8 Anisotropy of the Cosmic Microwave Background: Angular power spectrum of the CMB, incorporating all the pre-WMAP data (COBE, BOOMERanG, MAXIMA, DASI, CBI, ACBAR, FIRS, VSA, and other experiments). Variance of the multipole amplitude is plotted against multiple number; as indicated by the top scale, multipole $\ell$ measures the fluctuations on angular scale $\theta \sim 200^{\circ} / \ell$. Evidence of the baryon - photon oscillations can be seen as the distinct "acoustic peaks." The theoretical curve is the consensus cosmological model (image courtesy of C. Lineweaver). 


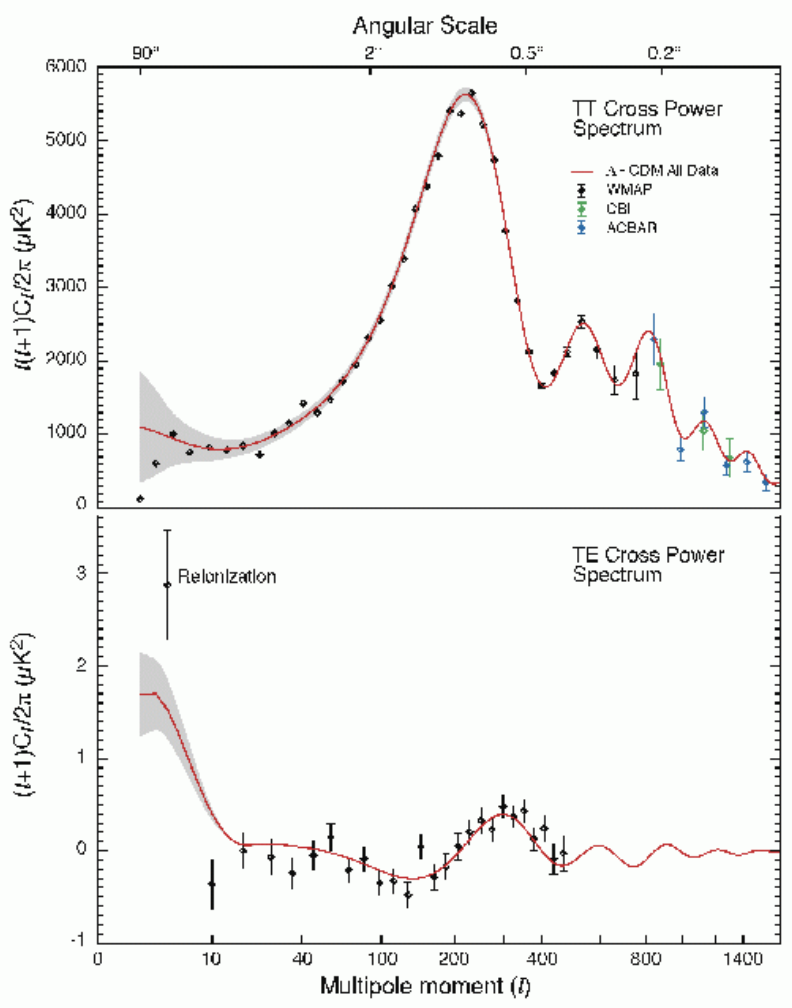

FIG. 9 Anisotropy of the Cosmic Microwave Background: The WMAP angular power spectrum (also includes data from CBI and ACBAR). The curve is the consensus cosmology model; the grey band includes cosmic variance. The WMAP measurements up to $\ell \sim 350$ are cosmic variance limited. The lower panel shows the anisotropy cross polarization power spectrum; the high point marked re-ionization is the evidence for re-ionization of the Universe at $z \sim 20$ (courtesy of NASA/WMAP Science Team). 


\section{Matter and Energy in the Universe: A Strange Recipe}

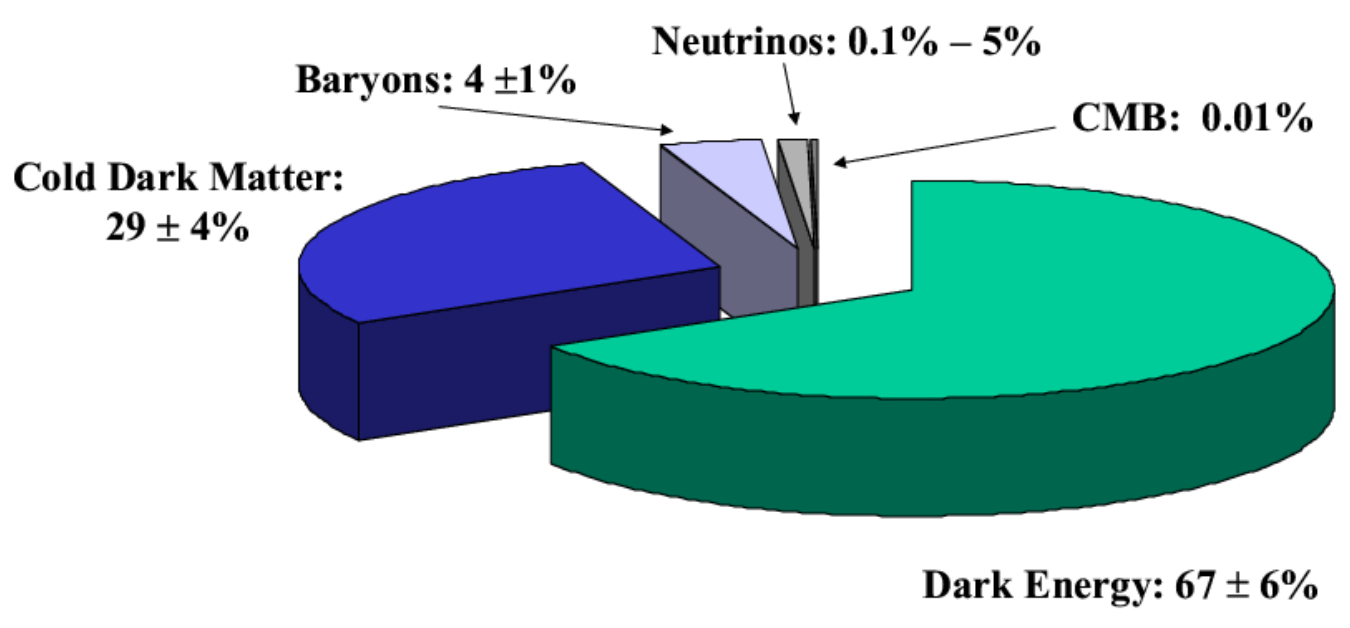

FIG. 10 The composition of the Universe today. Because the different components of the mass/energy budget evolve differently, the composition changes with time. For example, at very early times, photons and other relativitic particles were the dominant component; from 10,000 years until a few billion years ago, matter was the dominant component, and in the future dark energy will be the dominant component. 


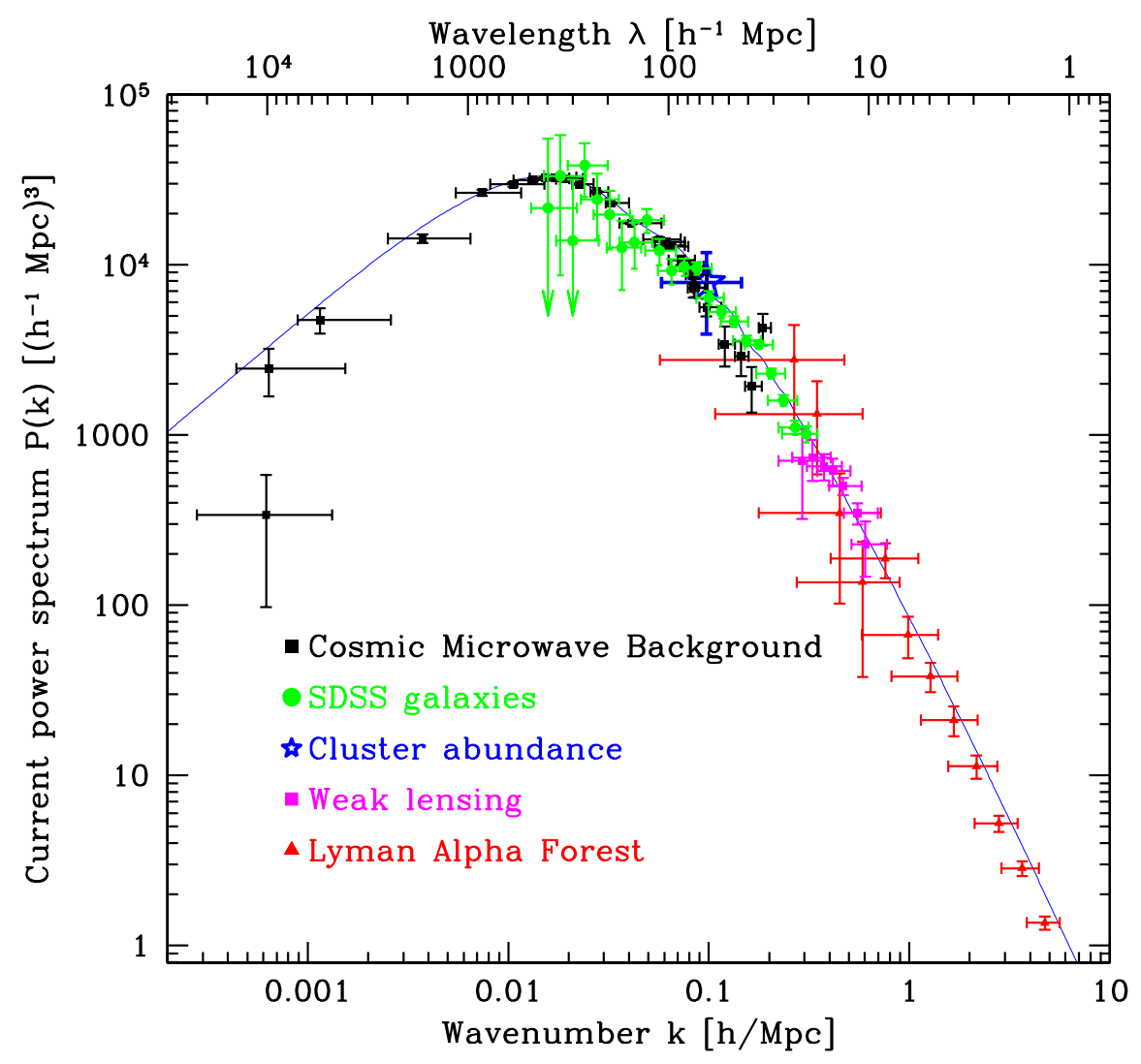

FIG. 11 Power spectrum of density inhomogeneity today obtained from a variety of measurements including large-scale structure, CMB, weak lensing, rich clusters and the Lyman-alpha forest. The curve is the theoretical prediction for the consensus cosmology model (from Tegmark et al., 2002). 
Tables

TABLE I OUR 16 COSMOLOGICAL PARAMETERS

\begin{tabular}{|c|c|c|c|}
\hline Parameter & Value ${ }^{a}$ & Description & $\mathrm{WMAP}^{b}$ \\
\hline & & Ten Global Parameters & \\
\hline$h$ & $0.72 \pm 0.07$ & Present expansion rate ${ }^{c}$ & $0.71_{-0.03}^{+0.04}$ \\
\hline$q_{0}$ & $-0.67 \pm 0.25$ & Deceleration parameter ${ }^{d}$ & $-0.66 \pm 0.10^{e}$ \\
\hline$t_{0}$ & $13 \pm 1.5 \mathrm{Gyr}$ & Age of the Universe ${ }^{f}$ & $13.7 \pm 0.2 \mathrm{Gyr}$ \\
\hline$T_{0}$ & $2.725 \pm 0.001 \mathrm{~K}$ & CMB temperature ${ }^{g}$ & \\
\hline$\Omega_{0}$ & $1.03 \pm 0.03$ & Density parameter ${ }^{h}$ & $1.02 \pm 0.02$ \\
\hline$\Omega_{\mathrm{B}}$ & $0.039 \pm 0.008$ & Baryon Density $^{i}$ & $0.044 \pm 0.004$ \\
\hline$\Omega_{\mathrm{CDM}}$ & $0.29 \pm 0.04$ & Cold Dark Matter Density ${ }^{i}$ & $0.23 \pm 0.04$ \\
\hline$\Omega_{\nu}$ & $0.001-0.05$ & Massive Neutrino Density ${ }^{j}$ & \\
\hline$\Omega_{X}$ & $0.67 \pm 0.06$ & Dark Energy Density ${ }^{i}$ & $0.73 \pm 0.04$ \\
\hline \multirow[t]{2}{*}{$w$} & $-1 \pm 0.2$ & Dark Energy Equation of State ${ }^{k}$ & $<-0.8(95 \% \mathrm{cl})$ \\
\hline & & Six Fluctuation Parameters & \\
\hline$\sqrt{S}$ & $5.6_{-1.0}^{+1.5} \times 10^{-6}$ & Density Perturbation Amplitude ${ }^{l}$ & \\
\hline$\sqrt{T}$ & $<\sqrt{S}$ & Gravity Wave Amplitude ${ }^{m}$ & $T<0.71 S(95 \% \mathrm{cl})$ \\
\hline$\sigma_{8}$ & $0.9 \pm 0.1$ & Mass fluctuations on $8 \mathrm{Mpc}^{n}$ & $0.84 \pm 0.04$ \\
\hline$n$ & $1.05 \pm 0.09$ & Scalar index ${ }^{h}$ & $0.93 \pm 0.03$ \\
\hline$n_{T}$ & - & Tensor index & \\
\hline$d n / d \ln k$ & $-0.02 \pm 0.04$ & Running of scalar index ${ }^{o}$ & $-0.03 \pm 0.02$ \\
\hline
\end{tabular}

qmile 1-o uncertainties quoted in this table represent our combined analysis of published data. Dentrett el ul., $2000 \mathrm{~s}$.

Freedman et al., 2001; note: $H_{0}=100 h \mathrm{~km} \mathrm{sec}^{-1} \mathrm{Mpc}^{-1}$.

${ }^{d}$ Supernovae results combined with measurements of the total matter density, $\Omega_{M}=\Omega_{\nu}+\Omega_{B}+$ $\Omega_{\mathrm{CDM}}$ and $\Omega_{0}$, assuming $w=-1$ (Perlmutter et al., 1999; Riess et al., 1998). 
${ }^{e}$ WMAP results (Bennett et al., 2003) combined with Tonrv et al., 2003.

fValue based upon CMB, globular cluster ages and current expansion rate (Knox et al., 2001;

Krauss and Chinabovel, 2002; Oswald et al., 1996).

sMather et al., 1999.

${ }^{h}$ Combined analysis of four CMB measurements (Sievers et al., 2002).

${ }^{i}$ Combined analysis of CMB, BBN, $H_{0}$ and cluster baryon fraction (Turner, 2002).

${ }^{j}$ Lower limit from SuperKamiokande measurements; upper limit from structure formation

(Elgarov et al., 2002; Fukuda et al., 2002).

${ }^{k}$ Supernova measurements, CMB and large-scale structure (Perlmutter et al., 1999).

${ }^{l}$ Contribution of density perturbations to the variance of the CMB quadrupole (with $T=0$ )

(Gorski et al., 1996).

${ }^{m}$ Contribution of gravity waves to the variance of the CMB quadrupole (upper limit)

(Kinnev et al., 2001).

${ }^{n}$ Variance in values reported is larger than the estimated errors; adopted error reflects this (Lahav et al., 2002).

${ }^{\circ}$ Deviation of the scalar perturbations from a pure power law (Lewis and Bridle, 2002). 